\title{
SARS-CoV-2 and myocardial injury: a role for Nox2?
}

\author{
Francesco Violi ${ }^{1}$. Daniele Pastori ${ }^{1} \cdot$ Pasquale Pignatelli $^{1} \cdot$ Roberto Cangemi $^{2}$
}

Received: 3 April 2020 / Accepted: 15 April 2020 / Published online: 12 May 2020

(c) Società Italiana di Medicina Interna (SIMI) 2020

\begin{abstract}
Severe acute respiratory syndrome coronavirus 2 (SARS-CoV-2) may be complicated by myocardial injury but at-risk patients as well as mechanism of disease are unclear. We gathered data regarding troponin levels in the so far reported SARS-CoV-2 patients and found a large variability in terms of troponin levels, patients with more severe disease, as those treated by ICU, presenting with higher percentage of troponin elevation. However, lack of prospective studies hampers adequate analysis of risk factors of myocardial damage. Previous study demonstrated that Nox2 is up-regulated in pneumonia and closely associated with troponin elevation suggesting Nox2 activation as mechanism eliciting myocardial damage; data in SARS-CoV-2 are still lacking. We hypothesize that SARS-Cov-2 may induce myocardial injury via Nox2-related ROS production and that analysis and eventually targeting Nox2 may be a novel approach to manage SARS-CoV-2.
\end{abstract}

The outbreak of pneumonia in Eastern and now in Europe and USA by severe acute respiratory syndrome coronavirus 2 (SARS-CoV-2) is a serious social issue for the high number of pulmonary complications needing intensive care units (ICU) treatment and for the mortality risk, which is particularly elevated in north Italy. Even if severe respiratory disease is a key factor precipitating poor outcome, other factors could also play a role. Previous study reported that community-acquired pneumonia (CAP) was associated with cardiovascular disease such as heart failure, myocardial infarction and atrial fibrillation and that myocardial damage, as depicted by elevated high-sensitivity cardiac troponin (hs-cTn) T, was detectable in $>50 \%$ of CAP patients; CAP severity was among the factors favoring myocardial injury [1]. As troponin elevation is associated with poor prognosis, we reviewed the data so far reported regarding the behavior of troponins in SARS-CoV-2 patients in relation to the disease severity.

We searched the PubMed database without language restriction for studies in human patients published between 2019 and present time, with a combination of text words

Francesco Violi

francesco.violi@uniroma1.it

1 Department of Clinical, Internal Medicine, Anesthesiologic and Cardiovascular Sciences, Sapienza University of Rome, Viale del Policlinico 155, 00161 Rome, Italy

2 Department of Translational and Precision Medicine, Sapienza University of Rome, Rome, Italy and Medical Subject Headings, including "troponin", "troponins", "myocardial injury", "laboratory", "Coronavirus", "Covid-19", "2019-nCOV”, "SARS-COV-2". Initially, 111 documents were evaluated. After excluding papers that did not report data on troponin levels, reviews, commentaries and letters, seven papers were selected, of which one was excluded because data about the prevalence of patients with troponin elevation were not expressly reported.

Of the six selected studies, five were set in China and one in USA and included a total number of 337 patients (Table 1). Myocardial injury was evaluated by cardiac troponin I (cTnI) in all but two, which measured hs-cTnI $[2,3]$. Myocardial injury (i.e., troponin levels exceeding the 99th percentile of the upper reference limit) was observed with a prevalence ranging from a minimum $2 \%$ to a maximum $44 \%$. In two studies, the prevalence of myocardial injury was compared between patients with severe conditions requiring ICU and patients not-requiring ICU, finding a significantly higher prevalence of troponin elevation in ICU patients. In particular, Wang et al. [4], in a population of 138 hospitalized patients for Covid-19-associated pneumonia, found that myocardial injury was present in $22 \%$ of ICU vs. $2 \%$ of nonICU patients $(p<0.001)$; similarly, Huang $C$ et al. [5], in a cohort of 41 patients, found a myocardial injury prevalence of $31 \%$ in ICU vs. $4 \%$ of non-ICU patients $(p=0.017)$. In three studies that reported data of ICU patients only [6, 7], prevalence of myocardial injury ranged between 14 and 44\%; while Liu et al. [2], in a cohort of 30 hospital infected workers, most of them without a severe pneumonia, showed a 
Table 1 Studies reporting myocardial injury during SARS-CoV-2 infection

\begin{tabular}{|c|c|c|c|c|c|c|c|c|}
\hline & $\mathrm{N}$ & Clinical characteristics & Age & Male (\%) & $\begin{array}{l}\text { Myocardial } \\
\text { injury }(\%)\end{array}$ & Non-ICU $(\%)$ & $\operatorname{ICU}(\%)$ & Mortality (\%) \\
\hline Wang et al. [4] & 138 & $\begin{array}{l}\text { Non-ICU: } 102 \\
\text { ICU: } 36\end{array}$ & $56(42-68)$ & 54.3 & 7.2 & 2 & 22 & 4.3 \\
\hline Yang et al. [6] & 52 & All critically ill ICU patients & $59.7 \pm 13.3$ & 67 & 23 & n.a & 23 & 61 \\
\hline Huang et al. [5] & 41 & $\begin{array}{l}\text { Non-ICU:28 } \\
\text { ICU: } 13\end{array}$ & $49(41-58)$ & 73 & 12 & 4 & 31 & 15 \\
\hline He et al. [7] & 54 & Severe/critical conditions & $68(60-74)$ & n.r & 44 & n.a & 44 & 48 \\
\hline Liu et al. [2] & 30 & $\begin{array}{l}\text { Medical workers, four with } \\
\text { severe conditions }\end{array}$ & $35 \pm 8$ & 33 & 16.7 & n.r & n.r & n.r \\
\hline Arentz et al. [3] & 21 & ICU admitted patients & $79(43-92)$ & 52 & 14 & n.r & 14 & 52.4 \\
\hline
\end{tabular}

n.a. not applicable, $n . r$. not reported

prevalence of myocardial injury of $16.7 \%$. Such large difference in terms of troponin elevation may depend on the time of analysis as troponin levels tend to increase by time after at admission; accordingly, in patients needed ICU treatment, Arentz et al. [3] reported troponin elevation at admission in $14 \%$ of patients, while the occurrence of cardiomyopathies was seen in a larger number (33\%) suggesting an even higher incidence of troponin elevation during the ICU treatment.

In ICU patients, myocardial injury may have an important impact on the prognosis of Covid-19-associated pneumonia: Yang et al. [6] found that mortality was $75 \%$ in patients with myocardial injury and $42 \%$ in patients without; $\mathrm{He} \mathrm{XW}$ et al. [7] reported an in-hospital mortality significantly higher in patients with myocardial injury than in patients without (61\% vs. $26 \%$, respectively, $p=0.013$ ).

Together these data indicate that myocardial injury is often detectable in SARS-CoV-2 patients with an impact much more evident according with disease severity; thus, the highest percentage of troponin elevation has been reported in patients needed ICU treatment. It is also of interest that patients with troponin elevation were at higher risk of mortality, but this potentially relevant association is biased by small sample size and lack of confounders analysis. Finally, the mechanisms accounting for myocardial injury in SARSCoV-2 was not investigated and, therefore, it is still unclear. Angiotensin converting enzyme 2 (ACE2) is a membranebound aminopeptidase, that is localized in the heart, vascular system and lung and is implicated in hypertension. Interestingly, ACE2 serves as functional receptor for SARS$\mathrm{CoV}-2$, that therefore, triggers lung infection upon binding its spike protein to ACE2 [8]. Such interaction may lead to ACE2 downregulation which is potentially deleterious for the heart as ACE2 serves to degrade angiotensin II to angiotensin 1-7, which counteract the angiotensin's vasoconstrictive activity [8]. Thus, ACE2 down-regulation by SARS-CoV-2 may be a potential trigger for oxidative stress as impaired angiotensin II degradation could lead to activation of Nox2, which is among the most important enzymes generating reactive oxidant species (ROS) [9](Fig. 1). Of note, Nox 2 localizes in the heart and rises during the acute phase of CAP [1], independently correlates with troponin elevation [1] and is associated with experimentally-induced heart failure; furthermore, Nox2 independently correlates with heart failure that often complicates CAP $[1,10]$. So far, Nox 2 has not been measured in SARS-CoV-2 patients; its evaluation might be useful to investigate the mechanism of disease of myocardial injury.

The study has limitation and implications. The study focused only in the Chinese population and included a small number of subjects. However, the myocardial injury is almost impressive and its impact with clinical outcomes should be studied prospectively. Due to the potentially already reported interplay between myocardial Nox2, cell injury and heart failure (Fig. 1) targeting Nox2 may be an approach to limit myocardial injury in patients affected by SARS-CoV-2. So far, however, there are not drugs in the market which inhibits Nox2; an alternative possibility is the interruption of the vicious circle Nox 2 activation -ROSNox2 activation, which perpetuates oxidative stress (Fig. 1). In this context, a simple and cheap approach may be provided by intravenous infusion of Vitamin $\mathrm{C}$ that behaves as an antioxidant in vivo only at millimolar concentrations. Intravenous infusion of $1 \mathrm{~g}$ ascorbic acid resulted in an antioxidant effect that ultimately down-regulate Nox2 activation without eliciting any side effect [11]. Of note, another drug commonly used for arthritis, i.e. tocilizumab, and now under investigation in SARS-CoV-2 patients, behaves also as an antioxidant and may interrupt the vicious circle Nox2-ROS-Nox2.

In conclusion, the data so far reported indicate that patients with SARS-CoV-2 display signs of myocardial injury, which is more evident in patients with severe disease. Prospective study is needed to assess the impact of myocardial injury on clinical outcomes. Nox 2 down-regulation may be an option to limit myocardial injury by SARS-CoV-2. 


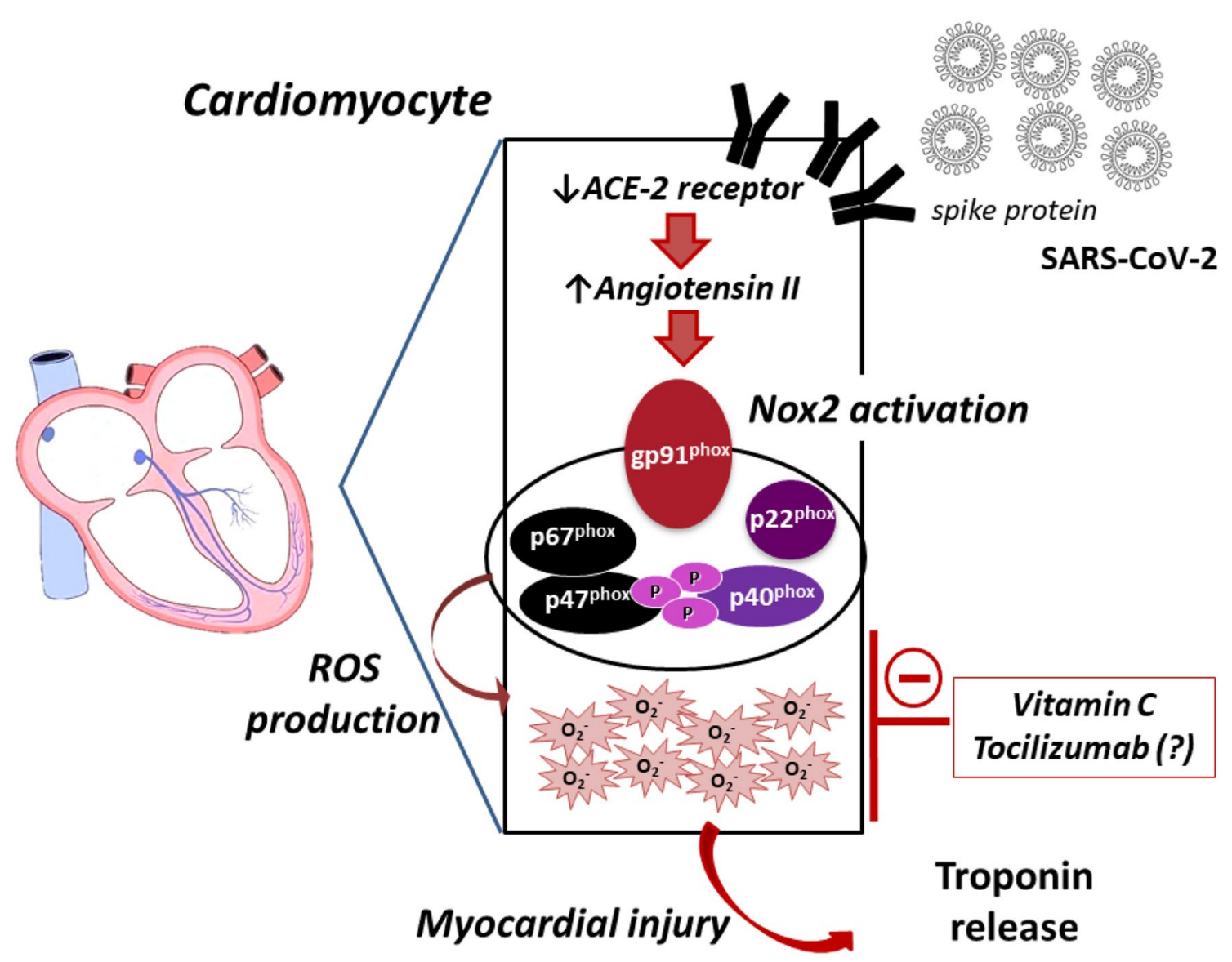

Fig. 1 Hypothetic mechanism accounting for oxidative stress by SARS-CoV-2. Interaction between SARS-CoV-2 and Nox2 in the heart. Interaction between SARS-CoV-2 and Angiotensin converting enzyme-2 (ACE-2) may lead to impaired angiotensin II degradation,

\section{Compliance with ethical standards}

Conflict of interest The authors declare no competing interests.

Statements of human and animal rights Not applicable.

Informed consent Not applicable.

\section{References}

1. Cangemi R, Calvieri C, Bucci T et al (2014) Is NOX2 upregulation implicated in myocardial injury in patients with pneumonia? Antioxid Redox Signal 20:2949-2954

2. Liu M, He P, Liu HG et al (2020) Clinical characteristics of 30 medical workers infected with new coronavirus pneumonia. Zhonghua Jie He He Hu Xi Za Zhi 43:209-214

3. Arentz M, Yim E, Klaff L, Lokhandwala S, Riedo FX, Chong M, Lee M (2020) Characteristics and outcomes of 21 critically subsequent Nox 2 activation and ultimately production of reactive oxidant species (ROS); ROS promote myocardial injury, an effect that may be blunted by Vitamin $\mathrm{C}$ or Tocilizumab

ill patients with COVID-19 in Washington State. JAMA. https ://doi.org/10.1001/jama.2020.4326

4. Wang D, Hu B, Hu C et al (2020) Clinical Characteristics of 138 Hospitalized Patients With 2019 Novel Coronavirus-Infected Pneumonia in Wuhan, China. JAMA 323:1061-1069

5. Huang C, Wang Y, Li X et al (2020) Clinical features of patients infected with 2019 novel coronavirus in Wuhan, China. Lancet 395:497-506

6. Yang X, Yu Y, Xu J et al (2020) Clinical course and outcomes of critically ill patients with SARS-CoV-2 pneumonia in Wuhan, China: a single-centered, retrospective, observational study. Lancet Respir Med. https://doi.org/10.1016/S2213 -2600(20)30079-5

7. He XW, Lai JS, Cheng J et al (2020) Impact of complicated myocardial injury on the clinical outcome of severe or critically ill COVID-19 patients. Zhonghua Xin Xue Guan Bing Za Zhi 48:E011

8. Clerkin KJ, Fried JA, Raikhelkar J et al (2020) Coronavirus disease 2019 (COVID-19) and cardiovascular disease. Circulation. https://doi.org/10.1161/CIRCULATIONAHA.120.046941

9. Khan Z, Shen XZ, Bernstein EA et al (2017) Angiotensin-converting enzyme enhances the oxidative response and bactericidal activity of neutrophils. Blood 130:328-339 
10. Cangemi R, Celestini A, Del Ben M et al (2014) Role of platelets in NOX2 activation mediated by TNFalpha in heart failure. Intern Emerg Med 9:179-185

11. Basili S, Tanzilli G, Mangieri E, Raparelli V, Di Santo S, Pignatelli P, Violi F (2010) Intravenous ascorbic acid infusion improves myocardial perfusion grade during elective percutaneous coronary intervention: relationship with oxidative stress markers. JACC Cardiovasc Interv 3:221-229
Publisher's Note Springer Nature remains neutral with regard to jurisdictional claims in published maps and institutional affiliations. 